\title{
HMGB1 Aggravates Pressure Overload-Induced Left Ventricular Dysfunction by Promoting Myocardial Fibrosis
}

\author{
Lei Zhang, ${ }^{1}$ Ying $\mathrm{Yu}^{2}{ }^{2}$ Peng Yu $\mathbb{D}^{\mathrm{D}},{ }^{1}$ Jian Wu, ${ }^{1}$ Aijun Sun, ${ }^{1}$ Yunzeng Zou, ${ }^{1}$ Yangang Su, ${ }^{1}$ \\ Hong Jiang $\mathbb{D}^{1}$, and Junbo Ge $\mathbb{D}^{1}$ \\ ${ }^{1}$ Department of Cardiology, Shanghai Institute of Cardiovascular Diseases, Zhongshan Hospital, \\ Shanghai Medical College of Fudan University, Shanghai 200032, China \\ ${ }^{2}$ Department of General Practice, Zhongshan Hospital, Shanghai Medical College of Fudan University, Shanghai 200032, China
}

Correspondence should be addressed to Hong Jiang; jiang.hong@zs-hospital.sh.cn

Received 14 May 2019; Accepted 12 May 2020; Published 17 June 2020

Academic Editor: Tomohiro Katsuya

Copyright (c) 2020 Lei Zhang et al. This is an open access article distributed under the Creative Commons Attribution License, which permits unrestricted use, distribution, and reproduction in any medium, provided the original work is properly cited.

Aim. Fibrosis had important effects on pressure overload-induced left ventricular (LV) dysfunction. High-mobility group box 1 (HMGB1), which was closely associated with fibrosis, was involved in the pressure overload-induced cardiac injury. This study determines the role of HMGB1 in LV dysfunction under pressure overload. Methods. Transverse aortic constriction (TAC) operation was performed on male C57BL/6J mice to build the model of pressure overload, while HMGB1 or PBS was injected into the LV wall. Cardiac function, collagen volume, and relevant genes were detected. Results. Echocardiography demonstrated that the levels of LV ejection fraction (LVEF) were markedly decreased on day 28 after TAC, which was consistent with raised collagen in the myocardium. Moreover, we found that the exposure of mice to TAC + HMGB1 is associated with higher mortality, BNP, and collagen volume in the myocardium and lower LVEF. In addition, real-time PCR showed that the expression of collagen type I, TGF- $\beta$, and MMP2 markedly increased in the myocardium after TAC, while HMGB1 overexpression further raised the TGF- $\beta$ expression but not collagen type I and MMP2 expressions. Conclusion. This study indicated that exogenous HMGB1 overexpression in the myocardium aggravated the pressure overload-induced LV dysfunction by promoting cardiac fibrosis, which may be mediated by increasing the TGF- $\beta$ expression.

\section{Introduction}

Heart failure has emerged as a major health problem during the past decades, but the underlying pathological mechanisms have not been fully disclosed. Myocardial fibrosis is involved in ventricular dysfunction in various heart diseases, such as myocardial infarction, cardiomyopathy, and hypertensive heart disease [1-3]. Meanwhile, pressure overload-induced myocardial fibrosis and subsequent heart failure are accompanied by inflammatory and fibrogenic responses [4-6].

High-mobility group box 1, a multifunctional cytokine, is involved in infection, injury, inflammation, and immune responses [7]. Emerging studies have shown that HMGB1 may be an important mediator in fibrosis not only in the liver and renal diseases $[8,9]$ but also in cardiovascular diseases [10-12] including experimental autoimmune myocarditis, postinfarction chronic heart failure, and diabetic cardiomyopathy. Combining these with our previous findings [13] that HMGB1 contributed to pressure overloadinduced cardiac hypertrophy and heart failure, the hypothesis seems reasonable in that HMGB1 may aggravate pressure overload-induced left ventricular dysfunction by mediating myocardial fibrosis. Our study, therefore, was to make the hypothesis testable.

\section{Materials and Methods}

2.1. Experimental Animals. Male wild-type C57BL/6J mice (8-10 weeks old, 22-24 g) were purchased from Shanghai Branch of National Rodent Laboratory Animal Resources, Shanghai, China. Mice were housed at $24 \pm 2^{\circ} \mathrm{C}$ under a 12 : $12 \mathrm{hr}$ light-dark cycle with ad libitum access to water and standard laboratory mouse chow [13]. All animal protocols 
were approved by the Animal Care Committee of Fudan University.

2.2. Surgical Interventions. The murine model of pressure overload was induced by transverse aortic constriction (TAC) $[14,15]$. The mice in the sham group were subjected to identical surgical interventions except for the constrictions in the aorta. As we described previously [13], $200 \mathrm{ng}$ of purified HMGB1 in $10 \mu \mathrm{l}$ PBS solution containing rhodamine spheres was injected before the ligation of the aortic arch. $2 \mu \mathrm{l}$ per injection (three injections in the anterior wall and two in the posterior wall) were administrated in the left ventricular (LV) wall. Successful injection was indicated by the presence of rhodamine in the site of injection.

2.3. Invasive Hemodynamic Assessment. Aortic blood pressure (ABP) and LV pressure (LVP) were evaluated before (baseline) surgery and on days 1, 3, 7, 14, and 28 after surgery, as we described previously $[15,16]$. Briefly, after anaesthesia, a micromanometer (Millar 1.4F, SPR 835; Millar Instruments, Houston, TX, USA) was carefully inserted through the right common carotid artery into LV. ABP, LVP, and systolic blood pressure (SBP) were obtained by the transducer connected to a power laboratory system (AD Instruments, Castle Hill, NSW, Australia).

2.4. Echocardiography Measurement. Echocardiographic analyses were performed with an animal-specific instrument (Vevo770; VisualSonics, Toronto, ON, Canada) before (baseline) TAC and on days 1, 3, 7, 14, and 28 after TAC. After inhalation anaesthesia of isoflurane, two-dimensional images were recorded in a parasternal long-axis projection with guided M-mode, as shown in our previous study [13]. The LV structure and systolic function including LV end-systolic pressure (LVESP), percent LV fractional shortening (LVFS \%), and ejection fraction (LVEF \%) were measured and averaged for 3-5 consecutive cardiac cycles as described previously [17]. All measurements were carried out by an experienced technician, who was unaware of the treatment of the mice.

2.5. Masson Staining. Mice were killed, and the whole heart was removed before (baseline) TAC and on days 1, 3, 7, 14, and 28 after TAC. Each heart was weighed and subsequently fixed with $4 \%$ paraformaldehyde $\left(4^{\circ} \mathrm{C}\right)$ for 12 hours. Paraffinembedded samples were sectioned at $5 \mu \mathrm{m}$ thickness and were stained with Masson Trichrome to evaluate fibrosis in the myocardium. The fibrotic lesions stained with blue were shown to be relative to the nonfibrotic lesions $(n=12)$ using scion image software.

2.6. RNA Extraction and Quantitative Real-Time Polymerase Chain Reaction (RT-PCR). According to the manufacturer's protocol, total RNA was extracted from LV tissues using the TRIzol reagent (Invitrogen, USA) and RT-PCR was performed with RT-PCR kits (TaKaRa, Kyoto, Japan). Specific primers for brain natriuretic peptide (BNP), collagen type 1,
TGF- $\beta$, MMP2, and $\beta$-actin are mentioned in Table 1 . The expression level of each gene was normalized to that of $\beta$-actin, which served as an endogenous internal control.

2.7. Statistical Analyses. All data were shown as mean\pm S.E.M and were analysed by one-way ANOVA followed by the post hoc least significant difference test using SPSS version 15.0 (SPSS Inc, Chicago, IL, USA). Values of $P<0.05$ were considered statistically significant.

\section{Results}

3.1. TAC-Induced LV Dysfunction and Myocardial Fibrosis. Echocardiographic assessment demonstrated that the levels of ABP, LVP, SBP, and LVESP were significantly increased after TAC $(P<0.01)$ (Figure 1$)$, while the levels of LVEF and LVFS were decreased, especially on day 28 after TAC $(P<0.01)$ (Figures 2(a) and 2(b)). These results showed that pressure overload-induced LV dysfunction models in mice were successfully built by TAC.

Masson staining showed that no significant collagen was detected on days $0,1,3,7$, and 14 after TAC; however, increased collagen size was observed at 4 weeks after TAC $(P<0.01)$ (Figure 2(c)), which was consistent with decreased $\mathrm{LV}$ function. These findings indicated that myocardial fibrosis was the key to pressure overload-induced LV dysfunction.

3.2. Exogenous HMGB1 Aggravated the Mortality of TAC Mice and Deteriorated the LV Dysfuction. To examine the roles of exogenous HMGB1 in the development of pressure overload-induced heart failure, exogenous HMGB1 was used to treat the mice with TAC. The mortality of mice was significantly increased within 28 days after TAC $(P<0.05)$. Moreover, the mortality of mice in TAC + HMGB1 was higher than that in TAC + PBS $(P<0.05)$.

We further assessed the effects of HMGB1 on LV structure and function of the mice at 4 weeks after TAC. Echocardiographic assessment demonstrated that HMGB1 failed to increase the levels of ABP, LVP, SBP, and LVESP of the TAC mice (Figure 3). However, the decreased LVEF and LVFS were markedly aggravated by cardiac injection of HMGB1 in mice with TAC $(P<0.05)$ (Figures $4(\mathrm{a})$ and $4(\mathrm{~b}))$. Additionally, real-time PCR showed that the BNP levels were significantly raised in the mice with TAC $(P<0.05)$. And, the TAC mice injected with exogenous HMGB1 had higher BNP levels in the myocardium than that of those injected with PBS (Figure 5(a)).

These findings indicated that exogenous HMGB1 significantly elevated the mortality of TAC mice and worsened the LV dysfunction.

3.3. HMGB1 Aggravated TAC-Induced Myocardium Fibrosis. Masson staining depicted that TAC significantly increased the collagen volume in interstitium and perivasculum area in the myocardium of the mice at 4 weeks after TAC; however, collagen volume in TAC + HMGB1 group was higher than that in the TAC+PBS group $(P<0.05)$. These results 
TABle 1: Primer sequences for quantitative real-time PCR.

\begin{tabular}{|c|c|}
\hline Gene & Forward $(\mathrm{F})$ and reverse $(\mathrm{R})$ primers \\
\hline $\mathrm{BNP}$ & F $5^{\prime}$ CCTGGCCCATCGCTTCT3' \\
\hline & R 5' CATCTGGGACAGCACCTTCA3' \\
\hline Collagen type 1 & F $5^{\prime}$ CTTCACCTACAGCACCCTTGTG3' \\
\hline TGF- $\beta 1$ & 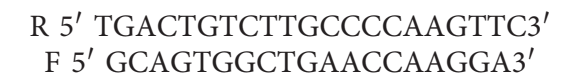 \\
\hline MMP2 & 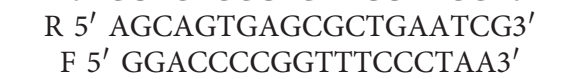 \\
\hline$\beta$-Actin & $\begin{array}{c}\text { R } 5^{\prime} \text { CAGGTTATCAGGGATGGCATTC3 } \\
\text { F } 5^{\prime} \text { CGATGCCCTGAGGCTCTTT3' } \\
\text { R } 5^{\prime} \text { TGGATGCCACAGGATTCCA3 }{ }^{\prime}\end{array}$ \\
\hline
\end{tabular}

BNP: brain natriuretic peptide; TGF- $\beta 1$ : transforming growth factor- $\beta 1$; MMP2: matrix metalloproteinase 2.

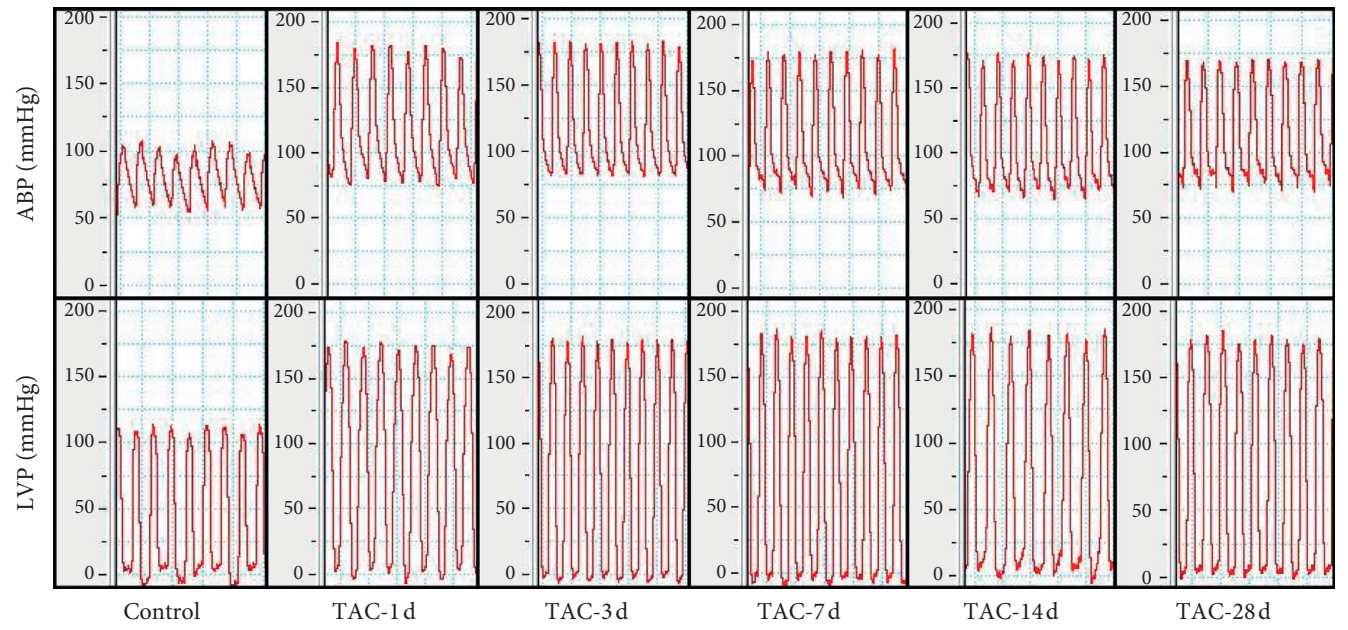

(a)

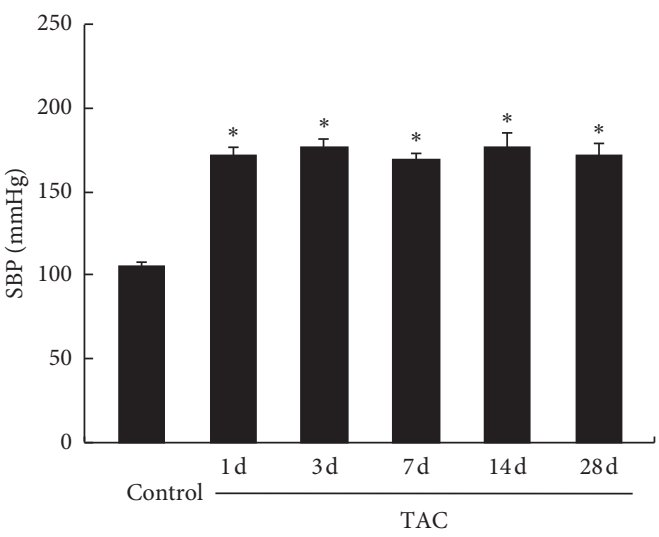

(b)

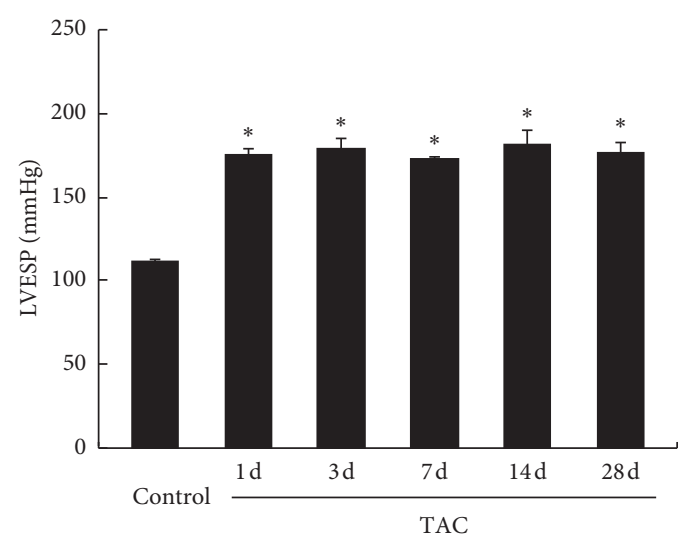

(c)

FIGURE 1: Haemodynamic assessment in mice within 4 weeks after transverse aortic constriction (TAC). ABP: aortic blood pressure; LVP: LV pressure; SBP: systolic blood pressure; LVESP: left ventricular end-systolic pressure. Values are expressed as mean \pm S.E.M. $n>8$ per group in each group. ${ }^{*} P<0.01$ versus control.

indicated that HMGB1 aggravated the TAC-induced ventricular fibrosis (Figure $4(\mathrm{c})$ ), resulting in a worsened ventricular function.

\subsection{Underlying Mechanisms in HMGB1-Induced Myocardial} Fibrosis. We further explored the potential mechanism of the HMGB1-mediated pressure overload-induced ventricular fibrosis. As we all know, collagen type I, TGF- $\beta$, and MMP2 were the important mediators in the development of fibrosis [18]. Therefore, we evaluated the effects of HMGB1 on these factors in TAC-induced myocardium fibrosis. We found that the levels of collagen type I, TGF- $\beta$, and MMP2 detected by real-time PCR were markedly increased in the mice with TAC $(P<0.05)$, while the expression of TGF- $\beta$ was further raised by the overexpression 


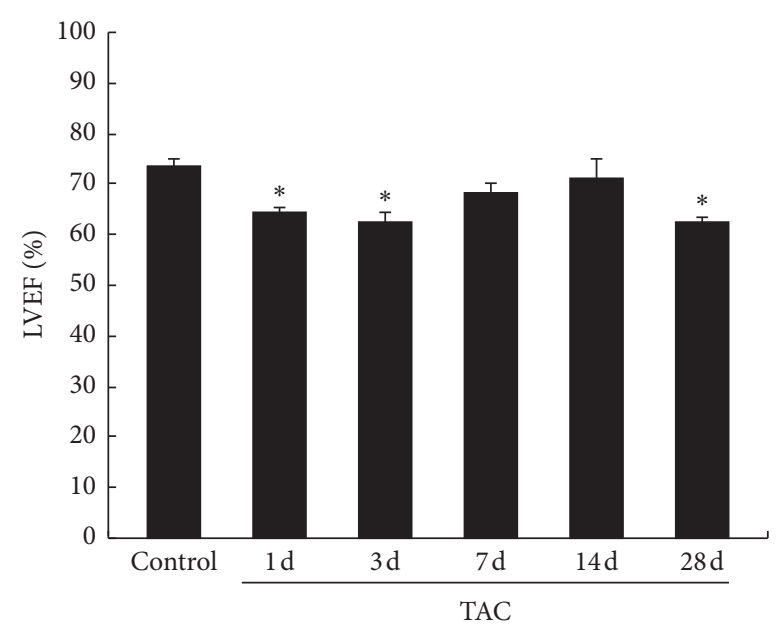

(a)
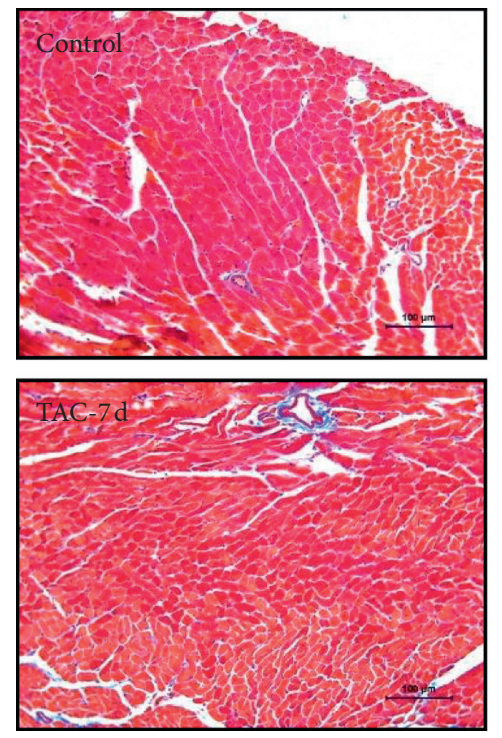

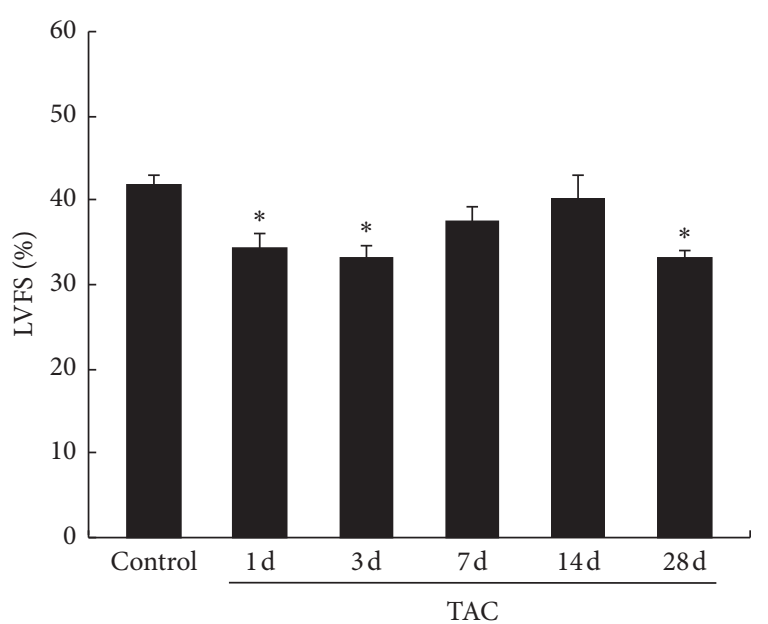

(b)
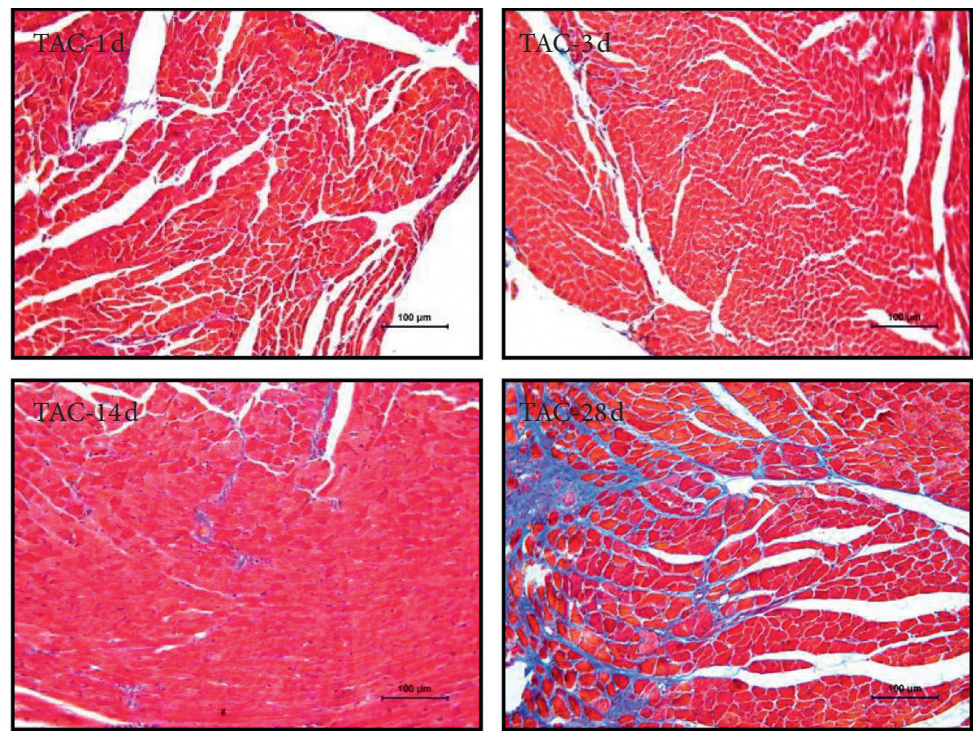

(c)

FIGURE 2: Echocardiography measurement and Masson staining in mice within 4 weeks after TAC measurement of LV ejection fraction (LVEF) and LV fractional shortening (LVFS). (c) Representative images of the whole hearts after TAC (scale bars $100 \mu \mathrm{m}$ ). Values are expressed as mean \pm S.E.M. $n>8$ per group in each group. ${ }^{*} P<0.01$ versus control.

of HMGB1 $(P<0.05)$. However, HMGB1 failed to upregulate the expression of collagen type I and MMP2 (Figures 5(b) and 5(d)). These data demonstrated that HMGB1 may aggravate the myocardial fibrosis under pressure overload via promoting the TGF- $\beta$ expression.

\section{Discussion}

In the present study, we demonstrated the effects of HMGB1mediated myocardial fibrosis on pressure overload-induced LV dysfunction.

HMGB1 was initially identified as a nonhistone DNAbinding nuclear protein that regulates multiple transcriptional factors to stabilize nucleosome [19], while extracellular HMGB1, as a novelty cytokine, is reported to mediate inflammation in the models of sepsis, autoimmune disease, acute hepatic necrosis, and acute lung injury [20-23].
Moreover, growing evidences support that HMGB1 is recognized as a critical factor in various heart diseases, including myocardial infarction and ischaemia-reperfusion injury. In addition, our study demonstrates that cardiac HMGB1 expression increases after TAC; meanwhile, exogenous HMGB1 aggravates TAC-induced cardiac hypertrophy, which is ameliorated by HMGB1 inhibition. In this study, we constructed murine pressure-overload model and furtherly overexpressed HMGB1 in the heart of mice. It was observed that the mortality of TAC mice was significantly increased by HMGB1 injection. Although HMGB1 failed to further increase the levels of ABP, LVP, SBP, and LVESP of the TAC mice, HMGB1 had significant negative effects on LVEF and LVFS. Similarly, it is reported that HMGB1 induced a negative inotropic effect on the left ventricle [24, 25]. However, some reports address that exogenous HMGB1 inhibits cardiac remodelling and improves the cardiac 


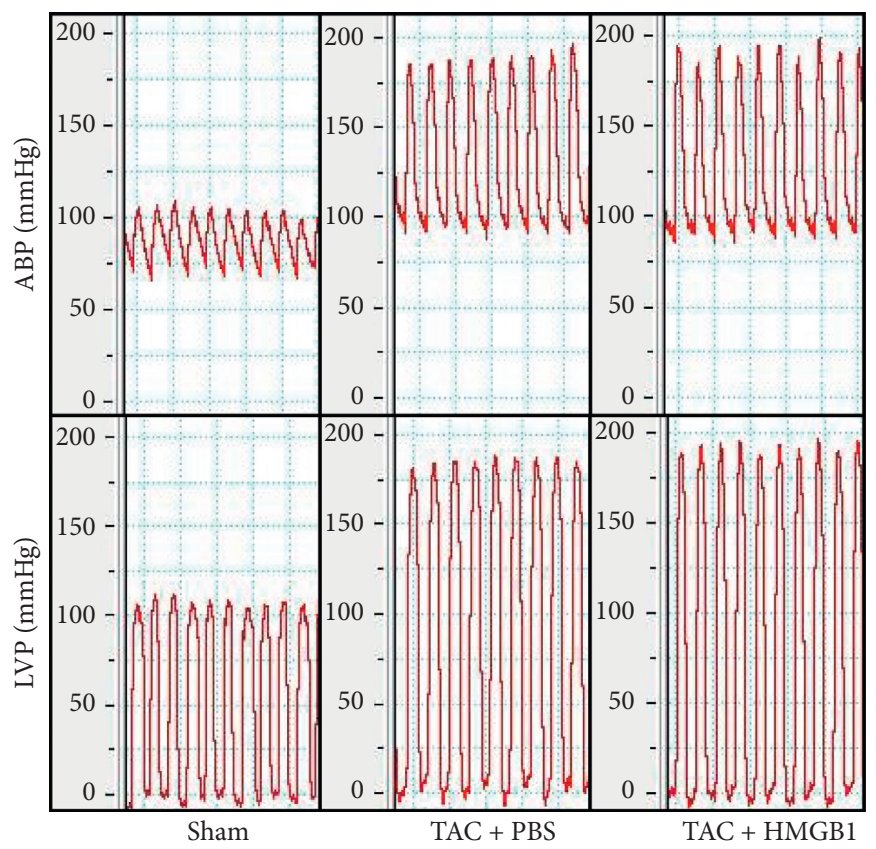

(a)

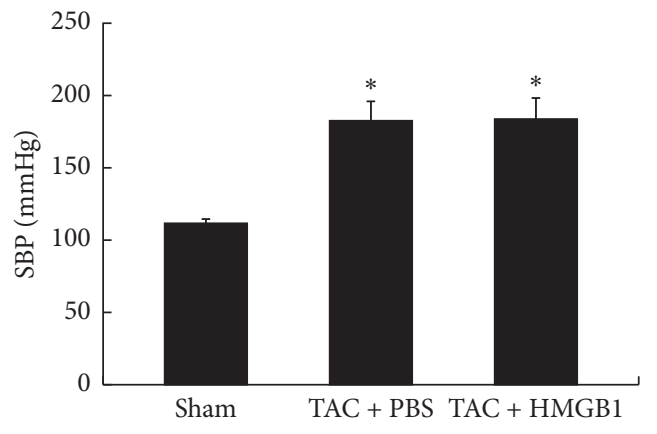

(b)

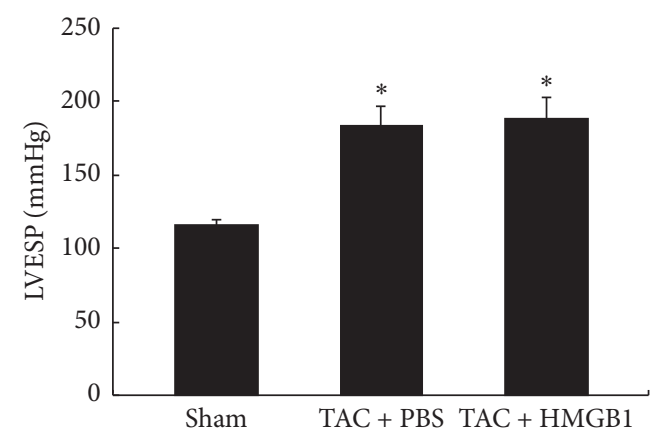

(c)

FIGURE 3: Haemodynamic assessment at 4 weeks after intervention. TAC: transverse aortic constriction; HMGB1: high-mobility group box 1; ABP: aortic blood pressure; LVP: LV pressure; SBP: systolic blood pressure; LVESP: left ventricular end-systolic pressure. Values are expressed as mean \pm S.E.M. $n>8$ per group in each group. ${ }^{*} P<0.01$ versus control.

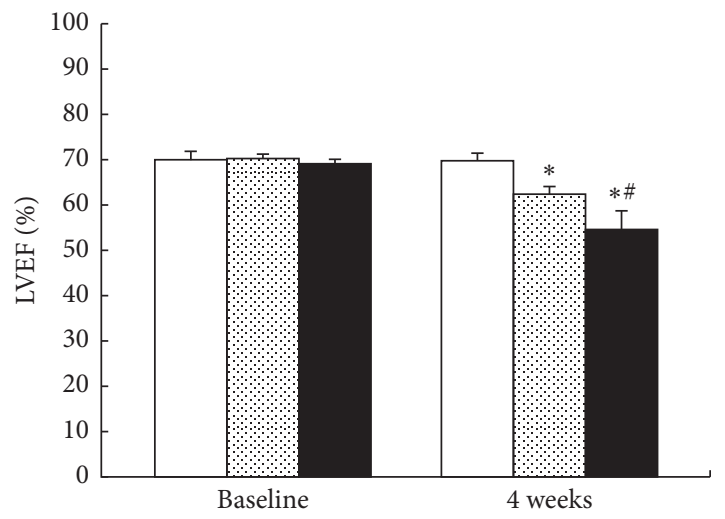

口 Sham

घ $\mathrm{TAC}+\mathrm{PBS}$

- TAC + HMGB1

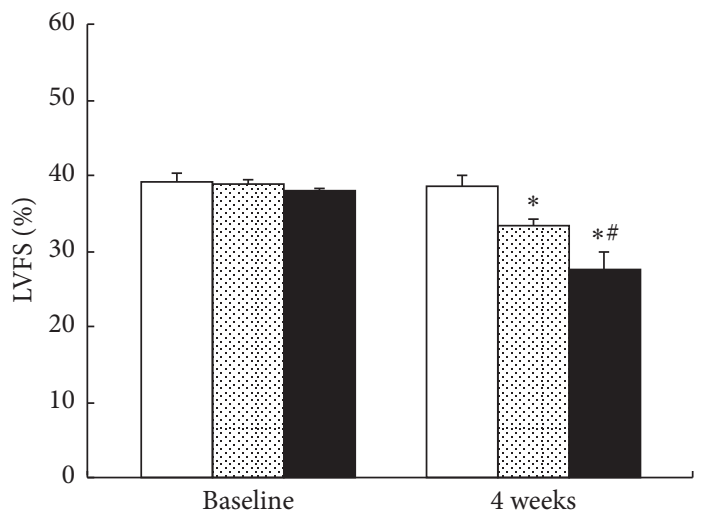

口 Sham

घ $\mathrm{TAC}+\mathrm{PBS}$

- $\mathrm{TAC}+$ HMGB1

(a)

Figure 4: Continued. 


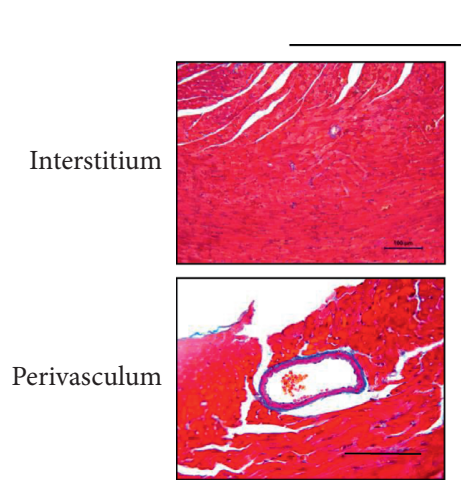

Sham
4 weeks

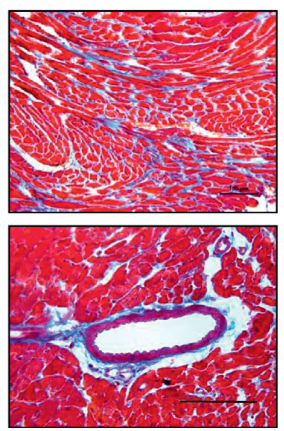

$\mathrm{TAC}+\mathrm{PBS}$
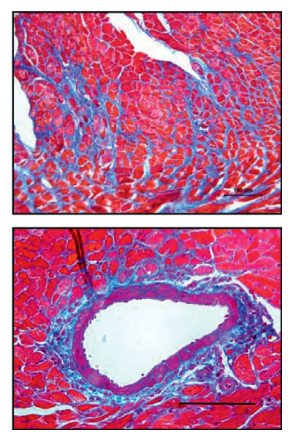

TAC + HMGB1 (c)

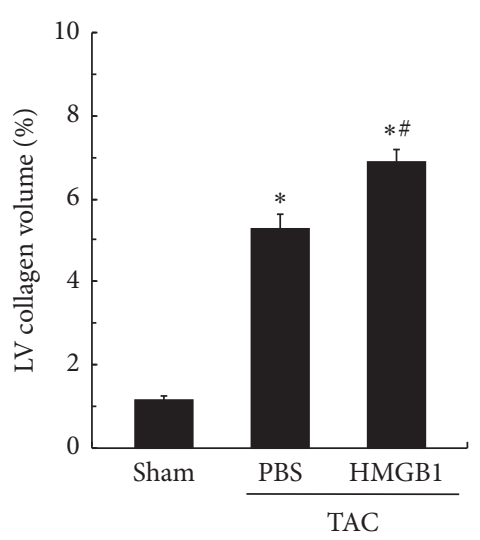

(d)

FiguRE 4: Echocardiography measurement and Masson staining at 4 weeks after intervention. (a, b) Echocardiography measurement of LV ejection fraction (LVEF) and LV fractional shortening (LVFS). TAC: transverse aortic constriction; HMGB1: high-mobility group box 1. (c) Representative images of the whole hearts after TAC (scale bars $100 \mu \mathrm{m}$ ). Values are expressed as mean \pm S.E.M. $n>8$ per group in each group. ${ }^{*} P<0.01$ versus control.

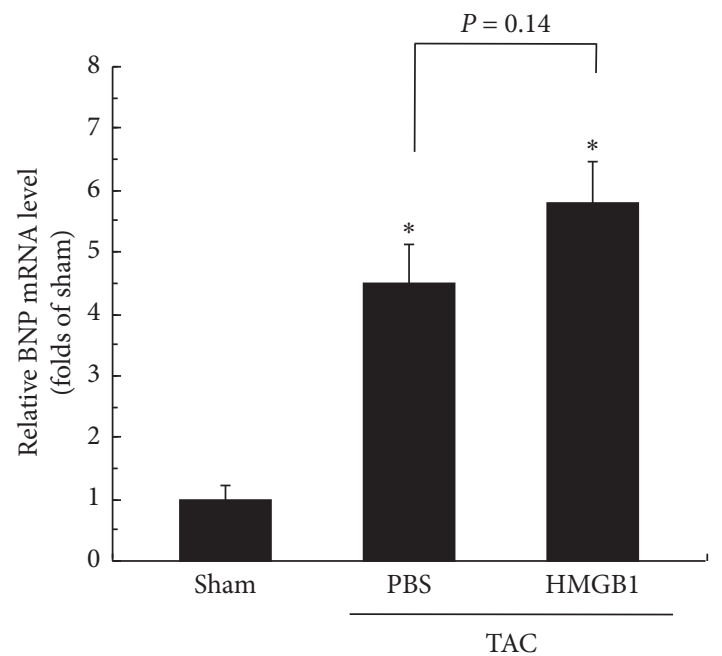

(a)

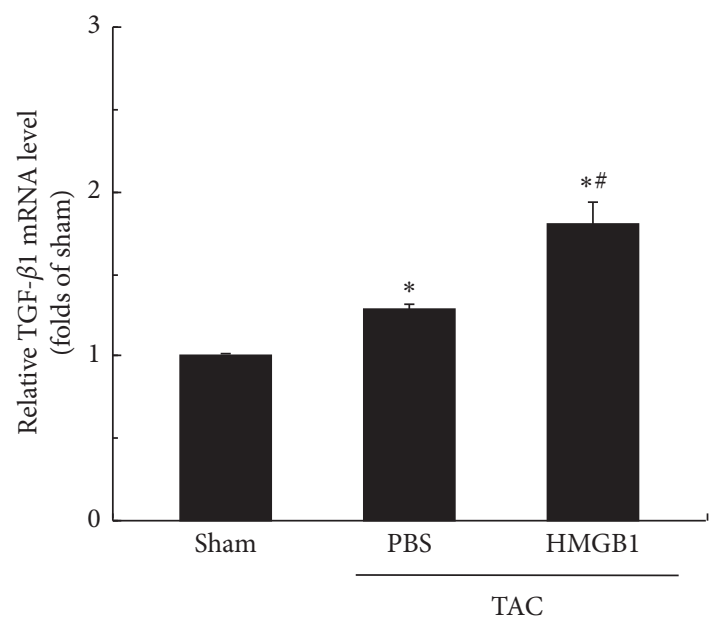

(c)

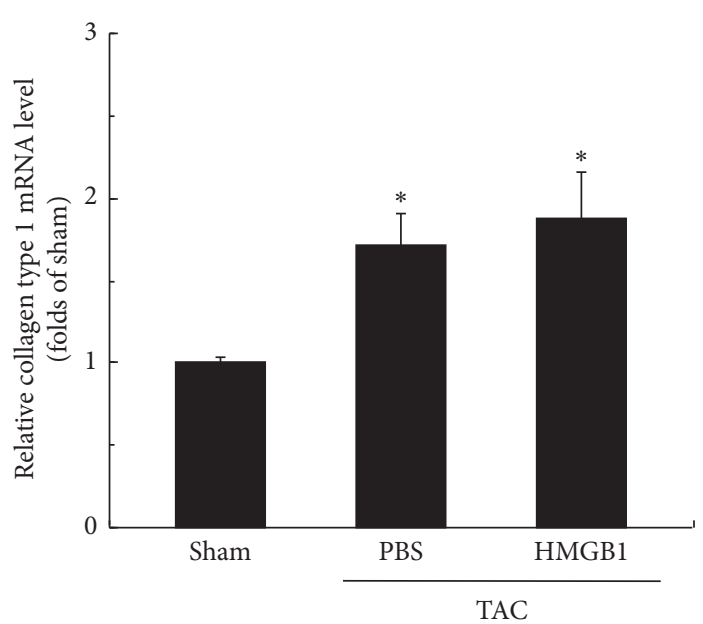

(b)

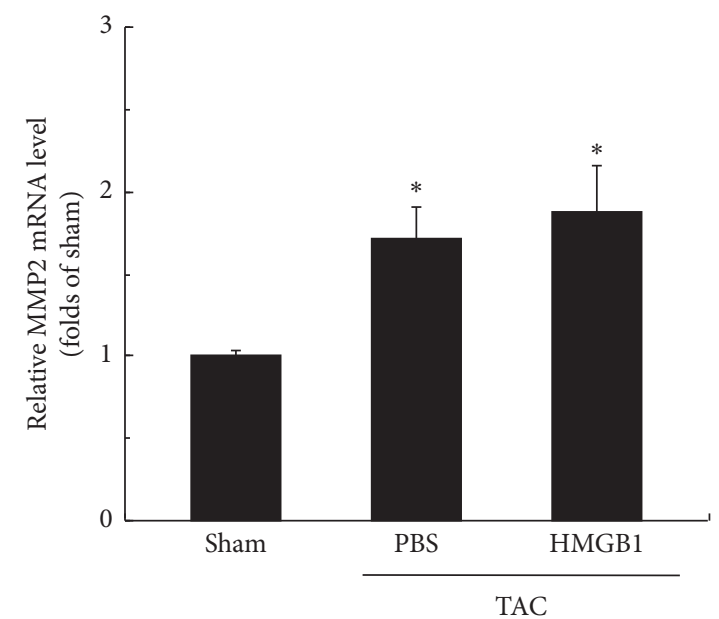

(d)

FIgURE 5: Quantitative real-Time PCR analysis of relevant mRNA expression in the myocardium at 4 weeks after intervention. TAC: transverse aortic constriction; HMGB1: high-mobility group box 1; BNP: brain natriuretic peptide. Data are represented as mean \pm S.E.M. $n>8$ per group in each group. ${ }^{*} P<0.05$ versus the sham group; ${ }^{\#} P<0.05$ versus the respective TAC + PBS group. 
function after myocardial infarction, which are not consistent with our results. We speculated that the reason that led to different conclusions was the different pathogenesis of the experimental models used in these investigations.

Previous studies suggested that inflammatory cytokines (e.g., TNF- $\alpha$ and IL-1 $\beta$ ), which were closely associated with collagen deposition in the myocardium, lead to myocardial fibrosis [26, 27]. Recent studies reported that HMGB1 played an important role in many tissue fibrosis including cardiac fibrosis [28-30]. A previous investigation showed that HMGB1 could directly lead to cardiac collagen deposition, which was associated with $\mathrm{PKC} \beta /$ Erk $1 / 2$ signalling pathway. Moreover, the activation of the TLRs/MyD88dependent signalling pathway was also involved in HMGB1mediated cardiac fibrosis [31]. In this study, we found that HMGB1 significantly increased the collagen volume and TGF- $\beta$ mRNA expression in the myocardium of TAC mice. Nevertheless, HMGB1 had no marked influences on the levels of collagen type I and MMP2 in the TAC mice.

\section{Conclusion}

This study showed that TAC contributed to a marked LV dysfunction, which was closely associated with myocardium fibrosis. Moreover, exogenous HMGB1 overexpression in myocardium aggravated the pressure overload-induced LV dysfunction by promoting cardiac fibrosis, which may be mediated by increasing the TGF- $\beta$ expression.

\section{Data Availability}

The data used to support the findings of this study are available from the corresponding author upon request.

\section{Conflicts of Interest}

The authors declared no conflicts of interest.

\section{Authors' Contributions}

Lei Zhang, Ying Yu and Peng Yu are equal contributors.

\section{Acknowledgments}

This work was supported by the National Natural Science Foundation of China (Nos. 81400193, 81671934, 81600294, 81670355, and 81521001).

\section{References}

[1] M. Yang, J. Xiong, Q. Zou, D.-D. Wang, and C.-X. Huang, "Chrysin attenuates interstitial fibrosis and improves cardiac function in a rat model of acute myocardial infarction," Journal of Molecular Histology, vol. 49, no. 6, pp. 555-565, 2018.

[2] T. H. Tsai, C. J. Lin, S. Chua et al., "Deletion of RasGRF1 attenuated interstitial fibrosis in streptozotocin-induced diabetic cardiomyopathy in mice through affecting inflammation and oxidative stress," International Journal of Molecular Sciences, vol. 19, no. 10, p. 3094, 2018.
[3] T. Huc, A. Drapala, M. Gawrys et al., "Chronic, low-dose TMAO treatment reduces diastolic dysfunction and heart fibrosis in hypertensive rats," American Journal of PhysiologyHeart and Circulatory Physiology, vol. 315, no. 6, pp. H1805-H1820, 2018.

[4] D. Levy, R. J. Garrison, D. D. Savage, W. B. Kannel, and W. P. Castelli, "Prognostic implications of echocardiographically determined left ventricular mass in the framingham heart study," New England Journal of Medicine, vol. 322, no. 22, pp. 1561-1566, 1990.

[5] J. J. Hunter and K. R. Chien, "Signaling pathways for cardiac hypertrophy and failure," New England Journal of Medicine, vol. 341, no. 17, pp. 1276-1283, 1999.

[6] J. D. Molkentin and G. W. Dorn, "Cytoplasmic signaling pathways that regulate cardiac hypertrophy," Annual Review of Physiology, vol. 63, no. 1, pp. 391-426, 2001.

[7] L. Ulloa and D. Messmer, "High-mobility group box 1 (HMGB1) protein: friend and foe," Cytokine \& Growth Factor Reviews, vol. 17, no. 3, pp. 189-201, 2006.

[8] A. R. R. Vicentino, V. C. Carneiro, D. Allonso et al., "Emerging role of HMGB1 in the pathogenesis of schistosomiasis liver fibrosis," Frontiers in Immunology, vol. 9, p. 1979, 2018.

[9] X. Chen, J. Ma, T. Kwan et al., "Blockade of HMGB1 attenuates diabetic nephropathy in mice," Scientific Reports, vol. 8, no. 1, p. 8319, 2018.

[10] Z. Su, J. Yin, T. Wang et al., "Up-regulated HMGB1 in EAM directly led to collagen deposition by a PKC $\beta /$ Erk1/2-dependent pathway: cardiac fibroblast/myofibroblast might be another source of HMGB1," Journal of Cellular and Molecular Medicine, vol. 18, no. 9, pp. 1740-1751, 2014.

[11] K. Takahashi, S. Fukushima, K. Yamahara et al., "Modulated inflammation by injection of high-mobility group box 1 recovers post-infarction chronically failing heart," Circulation, vol. 118, no. 14, pp. S106-S114, 2008.

[12] W.-k. Wang, B. Wang, Q.-h. Lu et al., "Inhibition of highmobility group box 1 improves myocardial fibrosis and dysfunction in diabetic cardiomyopathy," International Journal of Cardiology, vol. 172, no. 1, pp. 202-212, 2014.

[13] L. Zhang, M. Liu, H. Jiang et al., "Extracellular high-mobility group box 1 mediates pressure overload-induced cardiac hypertrophy and heart failure," Journal of Cellular and Molecular Medicine, vol. 20, no. 3, pp. 459-470, 2016.

[14] Y. Zou, Y. Liang, H. Gong et al., "Ryanodine receptor type 2 is required for the development of pressure overload-induced cardiac hypertrophy," Hypertension, vol. 58, no. 6, pp. 1099-1110, 2011.

[15] Y. Zou, J. Li, H. Ma et al., "Heat shock transcription factor 1 protects heart after pressure overload through promoting myocardial angiogenesis in male mice," Journal of Molecular and Cellular Cardiology, vol. 51, no. 5, pp. 821-829, 2011.

[16] J. You, J. Wu, G. Jiang et al., "Olmesartan attenuates cardiac remodeling through DLL4/Notch1 pathway activation in pressure overload mice," Journal of Cardiovascular Pharmacology, vol. 61, no. 2, pp. 142-151, 2013.

[17] S. Ling, Q. Sun, Y. Li et al., "CKIP-1 inhibits cardiac hypertrophy by regulating class II histone deacetylase phosphorylation through recruiting PP2A," Circulation, vol. 126, no. 25, pp. 3028-3040, 2012.

[18] D. Zhao, Y. Shi, Y. Dang, Y. Zhai, and X. Ye, "Daidzein stimulates collagen synthesis by activating the TGF- $\beta /$ smad signal pathway," Australasian Journal of Dermatology, vol. 56, no. 1, pp. e7-e14, 2015. 
[19] M. Stros, "HMGB proteins: interactions with DNA and chromatin," Biochimica et Biophysica Acta (BBA)-Gene Regulatory Mechanisms, vol. 1799, no. 1-2, pp. 101-113, 2010.

[20] H. Wang, O. Bloom, M. Zhang et al., "HMG-1 as a late mediator of endotoxin lethality in mice," Science, vol. 285, no. 5425, pp. 248-251, 1999.

[21] J. Sobajima, S. Ozaki, H. Uesugi et al., "Prevalence and characterization of perinuclear anti-neutrophil cytoplasmic antibodies (P-ANCA) directed against HMG1 and HMG2 in ulcerative colitis (UC)," Clinical and Experimental Immunology, vol. 111, no. 2, pp. 402-407, 1998.

[22] A. Tsung, R. Sahai, H. Tanaka et al., "The nuclear factor HMGB1 mediates hepatic injury after murine liver ischemiareperfusion," Journal of Experimental Medicine, vol. 201, no. 7, pp. 1135-1143, 2005.

[23] E. Abraham, J. Arcaroli, A. Carmody, H. wang, and K. J. Tracey, "Cutting edge: HMG-1 as a mediator of acute lung inflammation," The Journal of Immunology, vol. 165, no. 6, pp. 2950-2954, 2000.

[24] H.-P. Tzeng, J. Fan, J. G. Vallejo et al., "Negative inotropic effects of high-mobility group box 1 protein in isolated contracting cardiac myocytes," American Journal of Physiology-Heart and Circulatory Physiology, vol. 294, no. 3, pp. H1490-H1496, 2008.

[25] S. Hagiwara, H. Iwasaka, T. Uchino, and T. Noguchi, "High mobility group box 1 induces a negative inotropic effect on the left ventricle in an isolated rat heart model of septic shock," Circulation Journal, vol. 72, no. 6, pp. 1012-1017, 2008.

[26] K. J. Pulkki, "Cytokines and cardiomyocyte death," Annals of Medicine, vol. 29, no. 4, pp. 339-343, 1997.

[27] J. G. Abreu, N. I. Ketpura, B. Reversade, and E. M. De Robertis, "Connective-tissue growth factor (CTGF) modulates cell signalling by BMP and TGF- $\beta$," Nature Cell Biology, vol. 4, no. 8, pp. 599-604, 2002.

[28] M. Ebina, H. Taniguchi, T. Miyasho et al., "Gradual increase of high mobility group protein b1 in the lungs after the onset of acute exacerbation of idiopathic pulmonary fibrosis," Pulmonary Medicine, vol. 2011, Article ID 916486, 9 pages, 2011.

[29] A. Gaggar, S. M. Rowe, M. Hardision, and J. E. Blalock, "Proline-glycine-proline (PGP) and high mobility group box protein-1 (HMGB1): potential mediators of cystic fibrosis airway inflammation," The Open Respiratory Medicine Journal, vol. 4, no. 1, pp. 32-38, 2010.

[30] J. C. Leemans, L. M. Butter, W. P. Pulskens et al., "The role of Toll-like receptor 2 inflammation and fibrosis during progressive renal injury," PLoS One, vol. 4, no. 5, Article ID e5704, 2009

[31] P. Blyszczuk, G. Kania, T. Dieterle et al., "Myeloid differentiation factor-88/interleukin-1 signaling controls cardiac fibrosis and heart failure progression in inflammatory dilated cardiomyopathyflammatory dilated cardiomyopathy," Circulation Research, vol. 105, no. 9, pp. 912-920, 2009. 